\title{
PARTITIONS AND DIAMOND
}

\author{
PIERRE MATET
}

\begin{abstract}
We restate the diamond principle in terms of partitions, and we show that a weakening of diamond follows from the generalized continuum hypothesis.
\end{abstract}

For the duration of this paper $\kappa$ will denote a fixed regular uncountable cardinal. Our set-theoretic notation is that of $[\mathbf{4}]$.

For every ordinal $\alpha \leq \kappa$, let $(\kappa)^{\alpha}$ denote the collection of those sequences $X(\nu)$, $\nu<\alpha$, of pairwise disjoint nonempty subsets of $\kappa$ such that $\bigcup_{\nu<\alpha} X(\nu)=\kappa$ and $\bigcap X(\nu)<\bigcap X(\mu)$ whenever $\nu<\mu$. We recall that an ideal $I$ over $\kappa$ is $\kappa$-saturated if for every $X \in(\kappa)^{\kappa}$, there exists $\alpha<\kappa$ such that $X(\alpha) \in I$.

The following is an easy restatement of what is widely known as Ketonen's lemma (for a proof see Lemma 33.3 of [4]).

PROPOSITION 1. $\kappa^{<\kappa}=\kappa$ iff for every non- $\kappa$-saturated ideal I over $\kappa$, there exists a family $X_{\alpha} \in(\kappa)^{\kappa}, \alpha<2^{\kappa}$, such that for every $A \in\left[2^{\kappa}\right]^{<\kappa}$ and every $h \in \kappa^{A}, \bigcap_{\alpha \in A} X_{\alpha}(h(\alpha)) \notin I$.

Ketonen's lemma has applications in the theory of ultrafilters. One corollary states that if $\kappa$ is $2^{\kappa}$-compact and $H$ is a $\kappa$-complete filter over $\kappa$ such that its dual ideal is not $\kappa$-saturated, then there are $2^{2^{\kappa}} \kappa$-complete ultrafilters over $\kappa$ extending $H$. We omit the proof of the following, as it closely follows the proof of the related Theorem 3.2 of $[6]$.

COROLlaRY 2. Assume $\kappa^{<\kappa}=\kappa$, and let $H$ be a nontrivial filter over $\kappa$ such that its dual ideal is not $\kappa$-saturated. Then $H$ can be extended to an ultrafilter $K$ over $\kappa$ with the following property: If $p:[\kappa]^{<\kappa} \rightarrow K$ is a function such that $p(u) \supseteq p(w)$ whenever $u \subseteq w$, then there exists a function $q:[\kappa]^{<\kappa} \rightarrow K$ such that $q(u) \subseteq p(u)$ for every $u$, and that for every family $u_{\alpha}, \alpha<\gamma<\kappa$, of members of $[\kappa]^{<\kappa}, q\left(\bigcup_{\alpha<\gamma} u_{\alpha}\right)=\bigcap_{\alpha<\gamma} q\left(u_{\alpha}\right)$.

It is easy to modify the proof of Ketonen's lemma so as to obtain the following.

Proposition 3. $\kappa^{<\kappa}=\kappa$ iff there exists a family $X_{\alpha} \in(\kappa)^{\kappa}, \alpha<2^{\kappa}$, such that for every $A \in\left[2^{\kappa}\right]^{\kappa}$ and every $h \in \kappa^{A}$, there is an $E \in[\kappa]^{\kappa}$ with the property that $\left|E-X_{\alpha}(h(\alpha))\right|<\kappa$ for every $\alpha \in A$.

ProOF. Assume $\kappa^{<\kappa}=\kappa$. Choose a family $B_{\alpha}, \alpha<2^{\kappa}$, of members of $[\kappa]^{\kappa}$ such that $\left|B_{\alpha} \cap B_{\beta}\right|<\kappa$ whenever $\alpha \neq \beta$. For each $\alpha<2^{\kappa}$, let $f_{\alpha}$ be a mapping of $B_{\alpha}$ onto $\kappa$ such that $\left|\left\{\gamma \in B_{\alpha}: f_{\alpha}(\gamma)=\beta\right\}\right|=\kappa$ for every $\beta<\kappa$. Let $s_{\gamma}, \gamma<\kappa$, enumerate $[\kappa]^{<\kappa}$. For each $\alpha<2^{\kappa}$, define $g_{\alpha}: \kappa \rightarrow \kappa$ by letting $g_{\alpha}(\gamma)=f_{\alpha}(\beta)$ when

Received by the editors February 15, 1985.

1980 Mathematics Subject Classification. Primary 03E05, $03 E 50$.

Key words and phrases. Partitions, Ketonen's lemma, diamond. 
$s_{\gamma} \cap B_{\alpha}=\{\beta\}$, and $g_{\alpha}(\gamma)=0$ if $\left|s_{\gamma} \cap B_{\alpha}\right| \neq 1$. Also define $X_{\alpha} \in(\kappa)^{\kappa}$ by letting $\gamma, \delta$ lie in the same piece of $X_{\alpha}$ iff $g_{\alpha}(\gamma)=g_{\alpha}(\delta)$. Fix $A \in\left[2^{\kappa}\right]^{\kappa}$ and $h \in \kappa^{A}$. Let $k \in \kappa^{A}$ be such that $\gamma \in X_{\alpha}(h(\alpha))$ iff $g_{\alpha}(\gamma)=k(\alpha)$. Letting $a_{\beta}, \beta<\kappa$, enumerate $A$, define $t \in \kappa^{\kappa}$ as follows: Given $\gamma<\kappa$, for each $\beta<\gamma$ choose $\delta_{\beta} \in B_{a_{\beta}}$ such that $\delta_{\beta} \notin B_{a_{\alpha}}$ for $\alpha \in \gamma-\{\beta\}$, and $f_{a_{\beta}}\left(\delta_{\beta}\right)=k\left(a_{\beta}\right)$. Then pick $t(\gamma)$ so that $s_{t(\gamma)}=\left\{\delta_{\beta}: \beta<\gamma\right\}$. Finally put $E=\{t(\gamma): \gamma<\kappa\}$. Clearly, $\left|E-X_{\alpha}(h(\alpha))\right|<\kappa$ for every $\alpha \in A$.

Now for the other direction: Let $X_{\alpha} \in(\kappa)^{\kappa}, \alpha<2^{\kappa}$, be as in the statement of the proposition. It suffices to observe that for every cardinal $\mu<\kappa$, the family $\bigcap_{\alpha<\mu} X_{\alpha}(h(\alpha)), h \in \kappa^{\mu}$, consists of pairwise disjoint nonempty subsets of $\kappa$.

As formulated by Jensen, $\nabla_{\kappa}$ asserts the existence of a family $S_{\alpha}, \alpha<\kappa$, with each $S_{\alpha} \subseteq \alpha$ such that for any $A \subseteq \kappa$, the set $\left\{\alpha: A \cap \alpha=S_{\alpha}\right\}$ is stationary in $\kappa$. It is well known that $\nabla_{\kappa}$ is equivalent to the existence of functions $g_{\alpha}: \alpha \rightarrow \alpha, \alpha<\kappa$, such that for any $f: \kappa \rightarrow \kappa$, the set $\left\{\alpha: f \uparrow \alpha=g_{\alpha}\right\}$ is stationary. Also, Devlin [2] showed that $\mho_{\kappa}$ holds iff there are $S_{\alpha}, \alpha<\kappa$, with each $S_{\alpha} \subseteq \alpha$ such that for any $A \subseteq \kappa$, one can find $\alpha \geq \omega$ with $A \cap \alpha=S_{\alpha}$. Jensen [5] proved that $\vartheta_{\kappa}$ follows from $V=L$.

\section{PROPOSITION 4. The following are equivalent:}

(i) $\nabla_{\kappa}$.

(ii) There exists a family $X_{\alpha} \in(\kappa)^{\kappa}, \alpha<\kappa$, such that the diagonal intersection $\Delta\left\{X_{\alpha}(h(\alpha)): \alpha<\kappa\right\}$ is stationary for every $h \in \kappa^{\kappa}$.

(iii) There exists a family $X_{\alpha} \in(\kappa)^{\kappa}, \alpha<\kappa$, such that for every $h \in \kappa^{\kappa}$, there is a stationary $E \in[\kappa]^{\kappa}$ with the property that $\left|E-X_{\alpha}(h(\alpha))\right|<\kappa$ for every $\alpha<\kappa$.

(iv) There exists a family $Z_{\alpha} \in(\kappa)^{2}, \alpha<\kappa$, such that for every $h \in 2^{\kappa}$, there is a $\beta \geq \omega$ with $\beta \in \bigcap_{\alpha<\beta} Z_{\alpha}(h(\alpha))$.

PROOF. (i) $\rightarrow$ (ii): Choose functions $g_{\alpha}: \alpha \rightarrow \alpha, \alpha<\kappa$, such that for any $f: \kappa \rightarrow$ $\kappa$, the set $\left\{\alpha: f \mid \alpha=g_{\alpha}\right\}$ is stationary. For each $\alpha<\kappa$, put $A_{0}^{\alpha}=(\alpha+1) \cup\{\beta>$ $\left.\alpha: g_{\beta}(\alpha)=0\right\}$, and for each $\gamma>0, A_{\gamma}^{\alpha}=\left\{\beta>\alpha: g_{\beta}(\alpha)=\gamma\right\}$. Let $X_{\alpha}$ denote the partition of $\kappa$ into the pieces $A_{\gamma}^{\alpha}, \gamma<\kappa$. Fix $h \in \kappa^{\kappa}$, and let $f \in \kappa^{\kappa}$ be such that $X_{\alpha}(h(\alpha))=A_{f(\alpha)}^{\alpha}$ for every $\alpha<\kappa$. Set $E=\left\{\alpha: f \uparrow \alpha=g_{\alpha}\right\}$. Now it is easy to see that $\beta \in E$ iff $\beta \in X_{\alpha}(h(\alpha))$ for every $\alpha<\beta$. Thus $\Delta\left\{X_{\alpha}(h(\alpha)): \alpha<\kappa\right\}$ is stationary.

(ii) $\rightarrow$ (iii) is trivial.

(iii) $\rightarrow$ (iv): Pick a family $Z_{\alpha} \in(\kappa)^{2}, \alpha<\kappa$, such that for each $h \in 2^{\kappa}$, there is a stationary $E_{h} \in[\kappa]^{\kappa}$ with the property that $\left|E_{h}-Z_{\alpha}(h(\alpha))\right|<\kappa$ for every $\alpha<\kappa$. Fix $h \in 2^{\kappa}$. In case $E-\beta \subseteq \bigcap_{\alpha<\kappa} Z_{\alpha}(h(\alpha))$ for some $\beta$, there is nothing to prove, so assume otherwise. We define a function $k: E_{h} \rightarrow \kappa$ by letting $k(\beta)$ be the least $\alpha$ with $E-\beta \nsubseteq Z_{\alpha}(h(\alpha))$. If the set $\left\{\beta \in E_{h}: k(\beta)<\beta\right\}$ were stationary, then $k$ would be constant on some $H \in[E]^{\kappa}$, a contradiction. Hence, $\left\{\beta \in E_{h}: k(\beta) \geq \beta\right\}$ is stationary.

(iv) $\rightarrow$ (i): Pick a family $Z_{\alpha} \in(\kappa)^{2}, \alpha<\kappa$, as in (iv). For each $\beta<\kappa$, put $S_{\beta}=\left\{\alpha<\beta: \beta \in Z_{\alpha}(1)\right\}$. Given $A \subseteq \kappa$, define $h: \kappa \rightarrow 2$ by letting $h(\alpha)=1$ iff $\alpha \in A$. Choose $\beta \geq \omega$ with $\beta \in \bigcap_{\alpha<\beta} Z_{\alpha}(h(\alpha))$. Then for each $\alpha<\beta$,

$$
\alpha \in S_{\beta} \leftrightarrow \beta \in Z_{\alpha}(1) \leftrightarrow h(\alpha)=1 \leftrightarrow \alpha \in A .
$$

Thus $S_{\beta}=A \cap \beta$. 
Let $\lambda \geq \kappa$ be a fixed uncountable cardinal. The combinatorial principle $\diamond_{\kappa, \lambda}$ which was introduced by Jech in [3] asserts the existence of a family $S_{P}, P \in$ $[\lambda]^{<\kappa}$, with each $S_{P} \subseteq P$ such that for any $A \subseteq \lambda$, the set $\left\{P: A \cap P=S_{P}\right\}$ is stationary in $[\lambda]^{<\kappa}$. It is readily verified that $\diamond_{\kappa, \lambda}$ holds iff there are partitions $Z_{\alpha}$, $\alpha<\lambda$, of $[\lambda]<\kappa$ into two pieces $Z_{\alpha}(0)$ and $Z_{\alpha}(1)$ such that the diagonal intersection $\Delta\left\{Z_{\alpha}(h(\alpha)): \alpha<\lambda\right\}$ is stationary in $[\lambda]^{<\kappa}$ for every $h \in 2^{\lambda}$.

\section{REFERENCES}

1. F. G. Abramson, L. A. Harrington, E. M. Kleinberg and W. S. Zwicker, Flipping properties: A unifying thread in the theory of large cardinals, Ann. Math. Logic 12 (1977), 26-58.

2. K. J. Devlin, Variations on $\diamond$, J. Symbolic Logic 44 (1979), 51-58.

3. T. Jech, Some combinatorial problems concerning uncountable cardinals, Ann. Math. Logic 5 (1973), 165-198.

4. _ Set theory, Academic Press, New York, 1978.

5. R. B. Jensen, The fine structure of the constructible hierarchy, Ann. Math. Logic 4 (1972), 229-308.

6. K. Kunen, Ultrafilters and independent sets, Trans. Amer. Math. Soc. 172 (1972), 299-306.

8, RUe DE LA JUSSIENNE, 75002-PARIS, FranCE 